\title{
ON OPERATORS THAT ARE ALMOST ISOMETRIC ON THE POSITIVE CONES OF $L^{p}$-SPACES, $1<p<+\infty$
}

\author{
HUANG SENZHONG
}

(Communicated by Paul S. Muhly)

\begin{abstract}
A linear operator $T: L^{p}(\Omega) \rightarrow L^{p}\left(\Omega_{1}\right)$ is said to be almost isometric on the positive cone (a.i.p.c.) with distortion coefficient $\varepsilon$ if there is an $\varepsilon, 0 \leq \varepsilon<1$, such that $(1-\varepsilon)\|f\| \leq\|T f\| \leq\|f\|$ for all nonnegative functions $f$. We prove that if $p \in[2, \infty)$, then there are continuous functions $a_{p}(\cdot)$ and $b_{p}(\cdot)$ defined on $[0,1)$, with $a_{p}(0)=b_{p}(0)=1$, so that if $T: L^{p}(\Omega) \rightarrow L^{p}\left(\Omega_{1}\right)$ is an a.i.p.c. operator with distortion coefficient $\varepsilon$, then $a_{p}(\varepsilon)\|f\| \leq\|T f\| \leq b_{p}(\varepsilon)\|f\|$ for all $f \in L^{p}(\Omega)$. We also prove that such functions $a_{p}(\cdot)$ and $b_{p}(\cdot)$ exist for $p$ in the range $(1,2)$ if, in addition, $T$ is either positive or has dense range.
\end{abstract}

\section{INTRODUCTION}

In this paper, our notation is standard. By an $L^{p}$-space we mean a classical real space $L^{p}(\Omega, \mu)$ endowed with the usual $p$-norm, where $(\Omega, \mu)$ is a finite or infinite measure space. We write an element $f \in L^{p}(\Omega, \mu)$ as the sum of its positive part and negative part, i.e., we write $f=f^{+}-f^{-}$, where $f^{+}=f \vee 0$ and $f^{-}=(-f) \vee 0$. Moreover, for a measurable subset $\Omega_{0}$ of $\Omega$, we use the symbol $\|f\|_{\Omega_{0}}$ to stand for the value $\left(\int_{\Omega_{0}}|f|^{p} d \mu\right)^{1 / p}$.

We say that a linear operator $T: L^{p}(\Omega, \mu) \rightarrow L^{p}\left(\Omega_{1}, \mu_{1}\right)$ is almost isometric on the positive cone with distortion coefficient $\varepsilon, 0 \leq \varepsilon<1$, if

$$
(1-\varepsilon)\|f\| \leq\|T f\| \leq\|f\| \text { for all nonnegative } f \in L^{p}(\Omega, \mu) .
$$

We will abbreviate this by writing $T$ as an a.i.p.c. operator. We say that $T$ is isometric on the positive cone if we can take $\varepsilon=0$. In this case we write $T$ as an i.p.c. operator.

We use the following fact repeatedly: If $0<\alpha \leq 1$ and if $a, b \in \mathbf{R}$ then $|a+b|^{\alpha} \leq|a|^{\alpha}+|b|^{\alpha}$. This is often called by the subadditivity of $\alpha$-norm.

Received by the editors November 17, 1988.

1980 Mathematics Subject Classification (1985 Revision). Primary 47B99, 47D15.

Key words and phrases. Almost isometry, positive operator, modulus of convexity. 


\section{RESULT AND PROOF}

Proposition 1. Let $p \in[2, \infty)$ and $\varepsilon \in[0,1)$. Suppose that $T: L^{p}(\Omega) \rightarrow$ $L^{p}\left(\Omega_{1}\right)$ is an a.i.p.c. operator with distortion coefficient $\varepsilon$. Then

$$
a_{p}(\varepsilon)\|f\| \leq\|T f\| \leq b_{p}(\varepsilon)\|f\| \text { for all } f \in L^{p}(\Omega),
$$

where $a_{p}(\varepsilon)$ and $b_{p}(\varepsilon)$ are continuous functions depending only on $p$ and $\varepsilon$, and $a_{p}(0)=b_{p}(0)=1$.

To prove this proposition, two lemmas are needed.

Lemma 1. With the hypotheses of Proposition 1, let $f_{0} \in L^{p}(\Omega),\left\|f_{0}\right\| \leq 1$. Then

$$
\int_{\Omega_{1}}\left|T f_{0}^{ \pm}\right|^{p-2} \cdot\left|T f_{0}^{\mp}\right|^{2} \leq c_{p}(\varepsilon),
$$

where $c_{p}(\varepsilon)=\max \left(5\left[1-(1-\varepsilon)^{p}\right]^{(p-2) / 2 p},(p-1)\left[1-(1-\varepsilon)^{p}\right]^{1 / 6}\right)$.

Proof. Let $f=T f_{0}^{+}, g=T f_{0}^{-}$. Set

$$
H(t)=\|t f+g\|^{p}-\|g\|^{p}, \quad 0 \leq t \leq 1 .
$$

Fix $t, 0 \leq t \leq 1$. From the assumption that $T$ is an a.i.p.c. operator with distortion coefficient $\varepsilon$, we obtain

$$
(1-\varepsilon)\left\|t f_{0}^{+}+f_{0}^{-}\right\| \leq\|t f+g\| \leq\left\|t f_{0}^{+}+f_{0}^{-}\right\|
$$

and

$$
(1-\varepsilon)\left\|f_{0}^{-}\right\| \leq\|g\| \leq\left\|f_{0}^{-}\right\| .
$$

Thus

(1) $|H(t)| \leq \max \left(\left|\left\|t f_{0}^{+}+f_{0}^{-}\right\|^{p}-(1-\varepsilon)^{p}\left\|f_{0}^{-}\right\|^{p}\right|,\left|(1-\varepsilon)^{p}\left\|t f_{0}^{+}+f_{0}^{-}\right\|^{p}-\left\|f_{0}^{-}\right\|^{p}\right|\right)$.

We further have

$$
\begin{aligned}
\mid \| t f_{0}^{+} & +f_{0}^{-}\left\|^{p}-(1-\varepsilon)^{p}\right\| f_{0}^{-} \|^{p} \mid \\
& =\left|1-(1-\varepsilon)^{p}+\left[t^{p}-1+(1-\varepsilon)^{p}\right]\left\|f_{0}^{-}\right\|^{p}\right| \\
& \leq 1-(1-\varepsilon)^{p} \quad \text { for } 0 \leq t^{p} \leq 1-(1-\varepsilon)^{p}
\end{aligned}
$$

and similarly

$$
\left|(1-\varepsilon)^{p}\left\|t f_{0}^{+}+f_{0}^{-}\right\|^{p}-\left\|f_{0}^{-}\right\|^{p}\right| \leq 1-(1-\varepsilon)^{p} \quad \text { for } 0 \leq t \leq 1 .
$$

Combining (2) and (3) with (1), we find that

$$
|H(t)| \leq 1-(1-\varepsilon)^{p} \text { for all } 0 \leq t^{p} \leq 1-(1-\varepsilon)^{p} .
$$

Examining the second-order derivative of $H(t)$ we also have

$$
\begin{aligned}
0 \leq H^{\prime \prime}(t) & =p(p-1) \int_{\Omega_{1}}|t f+g|^{p-2} \cdot f^{2} \\
& \leq p(p-1)\|t f+g\|^{p-2}\|f\|^{2} \leq p(p-1) .
\end{aligned}
$$


(Here we have used, in order, Holder's inequality, the fact that $\|t f+g\| \leq$ $\left\|t f_{0}^{+}+f_{1}^{-}\right\| \leq 1$, and the assumption that $\|f\| \leq 1$.)

For fixed $t, 0<t^{p} \leq 1-(1-\varepsilon)^{p}$, choose $0<\xi<t$ and $0<\eta<\xi$ so that

$$
H^{\prime}(\xi) t=H(t)-H(0) \text { and } \quad H^{\prime \prime}(\eta) \xi=H^{\prime}(\xi)-H^{\prime}(0)
$$

Then

$$
\begin{aligned}
\left|H^{\prime}(0)\right| \leq\left|H^{\prime}(\xi)\right|+\left|H^{\prime}(\xi)-H^{\prime}(0)\right| & \leq t^{-1}|H(t)|+\left|H^{\prime \prime}(\eta) \xi\right| \\
& \leq t^{-1}\left[1-(1-\varepsilon)^{p}\right]+p(p-1) t
\end{aligned}
$$

(by (4) and (5)). In particular, by taking $t=\left[\frac{1-(1-\varepsilon)^{p}}{p(p-1)}\right]^{\frac{1}{2}}$, we obtain

$$
\left|H^{\prime}(0)\right| \leq 2\left[p(p-1)\left(1-(1-\varepsilon)^{p}\right)\right]^{\frac{1}{2}} \text {. }
$$

To continue the proof, we suppose $0<t^{p} \leq 1-(1-\varepsilon)^{p}$ again. For each such $t$, we use Taylor's formula to choose $0<\xi<t$ so that

$$
H(t)=H(0)+H^{\prime}(0) t+\frac{1}{2} H^{\prime \prime}(\xi) t^{2}
$$

Thus we have

(7)

$$
\begin{aligned}
\left|H^{\prime \prime}(\xi)\right| & \leq 2 t^{-2}\left[|H(T)|+\left|H^{\prime}(0)\right| t\right] \\
& \leq 2 t^{-2}\left\{1-(1-\varepsilon)^{p}+2\left[p(p-1) \cdot\left(1-(1-\varepsilon)^{p}\right)\right]^{\frac{1}{2}}+t\right\} \quad(\text { by }(4),(6)) \\
& \leq 6 t^{-2}[p(p-1)]^{\frac{1}{2}} \cdot\left[1-(1-\varepsilon)^{p}\right]^{\frac{1}{2}+\frac{1}{p}} \quad\left(\text { since } t^{p} \leq 1-(1-\varepsilon)^{p}\right) .
\end{aligned}
$$

Now two cases are considered: $p>3$ and $2 \leq p \leq 3$. For $p>3$, by examining the third-order derivative of $H(t)$ we have

$$
\begin{aligned}
\left|H^{\prime \prime \prime}(t)\right| & \leq p(p-1)(p-2) \int_{\Omega_{1}}|t f+g|^{p-3}|f|^{3} \\
& \leq p(p-1)(p-2)\|t f+g\|^{p-3}\|f\|^{3} \quad \text { (by Hölder's inequality) } \\
& \leq p(p-1)(p-2) \quad(\text { since }\|t f+g\| \leq 1 \text { and }\|f\| \leq 1)
\end{aligned}
$$

Next, choose $0<\eta<\xi$ so that

$$
H^{\prime \prime}(\xi)-H^{\prime \prime}(0)=H^{\prime \prime \prime}(\eta) \xi .
$$

From the above, we conclude that

$$
\begin{aligned}
H^{\prime \prime}(0) \leq & p(p-1)(p-2) t+\left|H^{\prime \prime}(\xi)\right| \\
\leq & 2 t^{-2}\left[1-(1-\varepsilon)^{p}\right]+2 t^{-1}\left[p(p-1) \cdot\left(1-(1-\varepsilon)^{p}\right)\right]^{\frac{1}{2}} \\
& +p(p-1)(p-2) t \quad(\text { by }(7))
\end{aligned}
$$

for all $0<t^{p} \leq 1-(1-\varepsilon)^{p}$. In particular, for $t_{0}=\left[1-(1-\varepsilon)^{p}\right]^{\alpha}$, where $\alpha=\frac{1}{4}$ if $p \geq 4$ and $\alpha=\frac{1}{p}$ if $3 \leq p<4$, after some straightforward computations, we finally obtain

$$
H^{\prime \prime}(0) \leq p(p-1)^{2}\left[1-(1-\varepsilon)^{p}\right]^{1 / 6} .
$$


For $2 \leq p \leq 3$, we conclude from the subadditivity of $(p-2)$-norm, that

$$
|| \xi f+\left.g\right|^{p-2}-\left.|g|^{p-2}|\leq| \xi f\right|^{p-2} \leq t^{p-2}|f|^{p-2} .
$$

And so

$$
\left|H^{\prime \prime}(\xi)-H^{\prime \prime}(0)\right| \leq p(p-1) t^{p-2} \int_{\Omega_{1}}|f|^{p} \leq p(p-1) t^{p-2} .
$$

Hence, by (7) again we find that

$$
H^{\prime \prime}(0) \leq p(p-1) t^{p-2}+6 t^{-2}[p(p-1)]^{\frac{1}{2}} \cdot\left[1-(1-\varepsilon)^{p}\right]^{\frac{1}{2}+\frac{1}{p}}
$$

for all $0<t^{p} \leq 1-(1-\varepsilon)^{p}$. In particular, for $t=\left[1-(1-\varepsilon)^{p}\right]^{1 / p}$, easy computations yield

$$
H^{\prime \prime}(0) \leq 5 p(p-1)\left[1-(1-\varepsilon)^{p}\right]^{(p-2) / 2 p} .
$$

This with $(8)$ yields

$$
\int_{\Omega_{1}}|g|^{p-2} \cdot f^{2}=H^{\prime \prime}(0) / p(p-1) \leq c_{p}(\varepsilon),
$$

where $c_{p}(\varepsilon)$ is defined in the lemma.

Interchanging $f$ and $g$ we also have

$$
\int_{\Omega_{1}}|f|^{p-2} \cdot g^{2} \leq c_{p}(\varepsilon)
$$

The proof is complete.

Lemma 2. For $p \in[2,+\infty)$ and real $a, b$ the inequality

$$
0 \leq|a+b|^{p}+|a-b|^{p}-2|a|^{p}-2|b|^{p} \leq M_{p}\left(a^{2}|b|^{p-2}+b^{2}|a|^{p-2}\right),
$$

holds, where $M_{p}=\frac{1}{2} p(p-1) \cdot K_{p}$ and $K_{p}$ is the maximum value of the function $K_{p}(t)=(1+t)^{p-2}+(1-t)^{p-2}-2 t^{p-2} \quad(0 \leq t \leq 1)$.

Sketch of the proof. The assertion (E1) is equivalent to this: For $0 \leq t \leq 1$,

$$
0 \leq(1+t)^{p}+(1-t)^{p}-2-2 t^{p} \leq M_{p}\left(t^{2}+t^{p-2}\right) .
$$

In order to prove $(*)$ we define two functions as follows:

$$
\begin{gathered}
h_{1}(t)=(1+t)^{p}+(1-t)^{p}-2-2 t^{p}, \quad 0 \leq t \leq 1, \\
h_{2}(t)=h_{1}(t)-M_{p} t^{2}, \quad 0 \leq t \leq 1 .
\end{gathered}
$$

It is easily seen that $h_{1}(t)$ is positive by examining its derivative. For the rest, note that

$$
h_{2}^{\prime \prime}(t)=p(p-1) \cdot\left[(1+t)^{p-2}+(1-t)^{p-2}-2 t^{p-2}\right]-2 \cdot M_{p} \leq 0,
$$

so $h_{2}^{\prime}(t) \leq h_{2}^{\prime}(0)=0$ for all $0 \leq t \leq 1$. Thus $h_{2}(t) \leq h_{2}(0)=0$ for all $0 \leq t \leq 1$.

This finishes the proof. 
Proof of Proposition 1. Suppose $f_{0} \in L^{p}(\Omega),\left\|f_{0}\right\| \leq 1$. Set

$$
f=T f_{0}^{+}, \quad g=T f_{0}^{-} .
$$

From the assumption that $T$ is an a.i.p.c. operator, we obtain

$$
(1-\varepsilon)\left\|f_{0}^{ \pm}\right\| \leq\left\|T f_{0}^{ \pm}\right\| \leq\left\|f_{0}^{ \pm}\right\| \text {and }(1-\varepsilon) \leq\|f+g\| \leq 1 .
$$

By Lemma 1 we also have

$$
\int_{\Omega_{1}}\left(f^{2}|g|^{p-2}+g^{2}|f|^{p-2}\right) \leq 2 c_{p}(\varepsilon) .
$$

Substituting $a=f$ and $b=g$ into the equality (E1) and then taking the integral, using (9) and (10), we find after some computations that

$$
a_{p}(\varepsilon)\left\|f_{0}\right\| \leq\left\|T f_{0}\right\| \leq b_{p}(\varepsilon)\left\|f_{0}\right\|,
$$

where $a_{p}(\varepsilon)=\left[\max \left(0,2(1-\varepsilon)^{p}-1\right)\right]^{1 / p}$ and $b_{p}(\varepsilon)=2-(1-\varepsilon)^{p}+2 M_{p} c_{p}(\varepsilon)$.

It is clear that $a_{p}(\varepsilon)$ and $b_{p}(\varepsilon)$ are continuous functions satisfying $a_{p}(0)=$ $b_{p}(0)=1$ (for $p=2$, note that $M_{2}=0$ ). This finishes the proof.

Recall that by definition a positive linear operator between two Banach lattices is a linear operator which maps positive elements to positive elements.

Proposition 2. Suppose $p \in(1,+\infty)$. Let $\varepsilon \in[0,1)$ and let $T: L^{p}(\Omega) \rightarrow$ $L^{p}\left(\Omega_{1}\right)$ be a positive a.i.p.c. operator with distortion coefficient $\varepsilon$. Then

$$
A_{p}(\varepsilon)\|f\| \leq\|T f\| \leq\|f\|
$$

for all $f \in L^{p}(\Omega)$, where $A_{p}(\varepsilon)=1-\varepsilon-\left[\frac{1-(1-\varepsilon)^{p}}{1-2^{1-p}}\right]^{1 / p}$.

Proof. Suppose $f \in L^{p}(\Omega)$. Since $T$ is positive, $T f^{ \pm} \geq 0$ and thus

$$
-T|f| \leq T f \leq T|f| \text {. }
$$

So we obtain

$$
\|T f\| \leq\|T|f|\| \leq\|f\| .
$$

By examining the function $h(t)=(1+t)^{p}-t^{p} \quad(t \geq 1)$ we get the following inequality:

$$
(a+b)^{p}-a^{p}-b^{p} \geq\left(2^{p}-2\right)(a \wedge b)^{p} \text { for all positive } a, b .
$$

Substituting $a=T f^{+}, b=T f^{-}$into (E2) and then integrating, we obtain

$$
\left(2^{p}-2\right)\left\|T f^{+} \wedge T f^{-}\right\|^{p} \leq\|T|f|\|^{p}-\left\|T f^{+}\right\|^{p}-\left\|T f^{-}\right\|^{p} .
$$

Since $T$ is an a.i.p.c. operator, this yields

$$
\left(2^{p}-2\right)\left\|T f^{+} \wedge T f^{-}\right\|^{p} \leq\|f\|^{p}-(1-\varepsilon)^{p}\|f\|^{p},
$$

or equivalently,

$$
\left\|T f^{+} \wedge T f^{-}\right\| \leq\left[\frac{1-(1-\varepsilon)^{p}}{2^{p}-2}\right]^{1 / p}\|f\|
$$


Note that $T f=T f-2 T f^{+} \wedge T f^{-}$, so we also have

$$
\|T f\| \geq\|T|f|\|-2\left\|T f^{+} \wedge T f^{-}\right\| .
$$

Combining these results we find that

$$
A_{p}(\varepsilon)\|f\| \leq\|T f\| \leq\|f\|,
$$

where $A_{p}(\varepsilon)$ is given above. This finishes the proof.

For the cases when $p \in(1,2)$, we require the notion of the modulus of convexity for $L^{p}$-spaces. Suppose $p \in(1,+\infty)$. Then there exists a nondecreasing continuous function $\varphi_{p}(\theta)$ defined on $[0,+\infty), \varphi_{p}(0)=0$, so that the following holds:

(11) For each $L^{p}$-space $E$ and $u \in E, u \neq 0$, if $v \in E^{*}$ satisfies $\|v\| \leq 1$ and $\langle v, u\rangle \geq(1-\theta)\|u\|$, then $\left\|v-g_{u}\right\| \varphi_{p}(\theta)$, where $g_{u}=\frac{|u|^{p-1} \operatorname{sign}(u)}{\|u\|^{p-1}}$.

Proposition 3. Let $p \in(1,2)$ and $\varepsilon_{p}^{\prime}=1-2^{(1-p) / p}$. Suppose that $T: L^{p}(\Omega) \rightarrow$ $L^{p}\left(\Omega_{1}\right)$ is an a.i.p.c. operator with distortion coefficient $\varepsilon$. If $0 \leq \varepsilon<\varepsilon_{p}$ and if the range of $T$ is dense in $L^{p}\left(\Omega_{1}\right)$, then $T$ is an onto-isomorphism such that

$$
B_{p}(\varepsilon)\|f\| \leq\|T f\| \leq(1+\varepsilon)\|f\|
$$

for all $f \in L^{p}(\Omega)$, where

$$
B_{p}(\varepsilon)=\max \left(\varepsilon_{p}-\varepsilon, 1-\varepsilon-\left[5\left(\varepsilon+\frac{\varphi_{p}(2 \varepsilon)}{\varepsilon_{p}-\varepsilon}\right)\right]^{1 /(p-1)}\right) .
$$

Proof. Suppose $f \in L^{p}(\Omega)$. Without loss of generality we may assume that $\|f\|=1$ and further that $\left\|f^{ \pm}\right\|>0$. We will achieve our goal in three steps.

Step 1. First we claim

$$
K_{\varepsilon} \leq\|T f\| \leq L_{\varepsilon},
$$

where $K_{\varepsilon}=2^{(p-1) / p}\left(\varepsilon_{p}-\varepsilon\right)$ and $L_{\varepsilon}=\left[2-(1-\varepsilon)^{p}\right]^{1 / p}$.

In fact, since $1<p<2$,

$$
|a+b|^{p}+|a-b|^{p} \leq 2|a|^{p}+2|b|^{p} \quad \text { for all } a, b \in \mathbf{R} \text {. }
$$

Therefore, by taking $a=T f^{+}, b=T f^{-}$and then integrating, this implies that

$$
\|T f\|^{p} \leq 2\left\|T f^{+}\right\|^{p}+2\left\|T f^{-}\right\|^{p}-\|T|f|\|^{p} .
$$

Since $T$ is an a.i.p.c. operator, we obtain from the above

$$
\|T f\| \leq L_{\varepsilon} .
$$

This is the right-hand side of (12). For the left-hand side, note that

$$
1=\|f\| \geq\left\|T f^{+}+T f^{-}\right\| \geq 2\left\|T f^{ \pm}\right\|-\|T f\| \geq 2(1-\varepsilon) \cdot\left\|f^{ \pm}\right\|-\|T f\|
$$

and from the fact that $\left\|f^{+}\right\|^{p}+\left\|f^{-}\right\|^{p}=\|f\|^{p}=1$, we have

$$
\|T f\| \geq 2(1-\varepsilon) \cdot \max \left(\left\|f^{+}\right\|,\left\|f^{-}\right\|\right)-1 \geq K_{\varepsilon} .
$$


More important, since the range of $T$ is dense in $L^{p}\left(\Omega_{1}\right)$, we conclude from (12) that $T$ is also an isomorphism from $L^{p}(\Omega)$ onto $L^{p}\left(\Omega_{1}\right)$. Thus, an easy computation shows that

$$
K_{\varepsilon}\|g\| \leq\left\|T^{*} g\right\| \leq L_{\varepsilon}\|g\| \text { for all } g \in L^{q}\left(\Omega_{1}\right),
$$

where $q=\frac{p}{p-1}$.

Step 2. In this step we will give an approximate result. To this end, put

$$
g_{|f|}=|T| f||^{p-1} \operatorname{sign}(T|f|) /\|T|f|\|^{p-1}
$$

and

$$
g_{f^{ \pm}}=\left|T f^{ \pm}\right|^{p-1} \operatorname{sign}\left(T f^{ \pm}\right) /\left\|T f^{ \pm}\right\|^{p-1} .
$$

Then they are three normalized elements of $L^{q}\left(\Omega_{1}\right)$ (the conjugate space of $\left.L^{p}\left(\Omega_{1}\right)\right)$, and satisfy

$$
\|T|f|\|=\left\langle g_{|f|}, T|f|\right\rangle \text { and }\left\|T f^{ \pm}\right\|=\left\langle g_{f^{ \pm}}, T f^{ \pm}\right\rangle .
$$

From these equations we have

$$
\left\langle|f|, L_{\varepsilon}^{-1} T^{*} g_{|f|}\right\rangle=L_{\varepsilon}^{-1}\left\langle T|f|, g_{|f|}\right\rangle=L_{\varepsilon}^{-1}\|T|f|\| \geq L_{\varepsilon}^{-1}(1-\varepsilon)
$$

and similarly

Since

$$
\left\langle f^{ \pm}, L_{\varepsilon}^{-1} T^{*} g_{f^{ \pm}}\right\rangle \geq L_{\varepsilon}^{-1}(1-\varepsilon) .
$$

$$
\left\|L^{-1} T^{*} g_{|f|}\right\| \leq L_{\varepsilon}^{-1} L_{\varepsilon}\left\|g_{|f|}\right\|=1 \text { and }\left\|L_{\varepsilon}^{-1} T^{*} g_{f^{ \pm}}\right\| \leq L_{\varepsilon}^{-1} L_{\varepsilon}\left\|g_{f^{ \pm}}\right\|=1
$$

(by (13)), we find that

$$
\left\|L_{\varepsilon}^{-1} T^{*} g_{|f|}-|f|^{p-1} /\right\| f\left\|^{p-1}\right\| \leq \varphi_{p}\left(1-\frac{1-\varepsilon}{L_{\varepsilon}}\right)
$$

and

$$
\left\|L_{\varepsilon}^{-1} T^{*} g_{f^{ \pm}}-\left(f^{ \pm}\right)^{p-1} /\right\| f^{ \pm}\left\|^{p-1}\right\| \leq \varphi_{p}\left(1-\frac{1-\varepsilon}{L_{\varepsilon}}\right) \quad \text { (by using (11)). }
$$

Now, by using the relation that $|f|^{p-1}=\left(f^{+}\right)^{p-1}+\left(f^{-}\right)^{p-1}$, from above we also have

$$
\left\|T^{*}\left(\|f\|^{p-1} \cdot g_{|f|}-\left\|f^{+}\right\|^{p-1} \cdot g_{f^{+}}-\left\|f^{-}\right\|^{p-1} \cdot g_{f^{-}}\right)\right\| \leq 3 L_{\varepsilon} \varphi_{p}\left(1-\frac{1-\varepsilon}{L_{\varepsilon}}\right) .
$$

By (13), this implies that

$$
\|\| f\left\|^{p-1} \cdot g_{|f|}-\right\| f^{+}\left\|^{p-1} \cdot g_{f^{+}}-\right\| f^{-}\left\|^{p-1} \cdot g_{f^{-}}\right\| \leq \frac{3 L_{\varepsilon}}{K_{\varepsilon}} \cdot \varphi_{p}\left(1-\frac{1-\varepsilon}{L_{\varepsilon}}\right) \text {. }
$$

Together with the definitions of $g_{|f|}, g_{f^{ \pm}}$, and $T$ this also implies that

(17) $\||T| f\|^{p-1} \cdot \operatorname{sign}(T|f|)-\left|T f^{+}\right|^{p-1} \cdot \operatorname{sign}\left(T f^{+}\right)-\left|T f^{-}\right|^{p-1} \cdot \operatorname{sign}\left(T f^{-}\right) \|$ $\leq M_{\varepsilon}$, where

(18) $M_{\varepsilon}=3\left[1-(1-\varepsilon)^{p-1}\right]+\frac{3 L_{\varepsilon}}{K_{\varepsilon}} \cdot \varphi_{p}\left(1-\frac{1-\varepsilon}{L_{\varepsilon}}\right)$. 
Finally, to complete the proof we set

$$
\Omega_{0}=\left\{\omega \in \Omega_{1}: T f^{+}(\omega) \cdot T f^{-}(\omega) \geq 0\right\} .
$$

Then (17) implies that

$$
\left\|\left(\left|T f^{+}\right|+\left|T f^{-}\right|\right)^{p-1}-\left|T f^{+}\right|^{p-1}-\left|T f^{-}\right|^{p-1}\right\|_{\Omega_{0}} \leq M_{\varepsilon},
$$

where the norm is the $q$-norm. Now, for $1<p<2$ and positive $a$, $b$, we have

$$
a^{p-1}+b^{p-1}-(a+b)^{p-1} \geq\left(2-2^{p-1}\right) \cdot(a \wedge b)^{p-1} .
$$

(This is easily checked by considering the function $g(t)=t^{p-1}-(1+t)^{p-1}$ for $t \geq 1$.) So by substituting $a=\left|T f^{+}\right|$and $b=\left|T f^{-}\right|$into it and then integrating on the subset $\Omega_{0}$, we obtain

$$
\left\|\left|T f^{+}\right| \wedge\left|T f^{-}\right|\right\|_{\Omega_{0}} \leq\left[\frac{M_{\varepsilon}}{2-2^{p-1}}\right]^{1 /(p-1)} \text {, after some computations using (19). }
$$

Since $|T f| \geq|T| f||$ on $\Omega_{1} \backslash \Omega_{0}$, we have

$$
\begin{aligned}
\|T f\| & =\|T f\|_{\Omega_{1} \backslash \Omega_{0}}+\|T f\|_{\Omega_{0}} \\
& \geq\|T|f|\|_{\Omega_{1} \backslash \Omega_{0}}+\left\|T f^{+}|-| T f^{-} \mid\right\|_{\Omega_{0}} \\
& \geq\|T|f|\|_{\Omega_{1} \backslash \Omega_{0}}+\|T|f|\|_{\Omega_{0}}-2\left\|T f^{+}|\wedge| T f^{-} \mid\right\|_{\Omega_{0}} \\
& \geq 1-\varepsilon-\left[\frac{M_{\varepsilon}}{2^{2-p}-1}\right]^{1 /(p-1)} \quad(\text { by }(20) \text { and }\|T|f|\| \geq(1-\varepsilon)) .
\end{aligned}
$$

Combining the inequalities obtained above, we conclude that

$$
\left\{1-\varepsilon-\left[\frac{M_{\varepsilon}}{2^{2-p}-1}\right]^{1 /(p-1)}\right\} \cdot\|f\| \leq\|T f\| \leq L_{\varepsilon}\|f\| .
$$

Step 3. In order to complete the proof, some computations are needed. To save space we summarize them below:

$$
\begin{gathered}
L_{\varepsilon} \leq 1+\varepsilon \text { and } K_{\varepsilon} \geq \varepsilon_{p}-\varepsilon ; \\
\varphi_{p}\left(1-\frac{1-\varepsilon}{L_{\varepsilon}}\right) \leq \varphi_{p}\left(1-\frac{1-\varepsilon}{1+\varepsilon}\right) \leq \varphi_{p}(2 \varepsilon)\left(\text { since } \varphi_{p}(\cdot) \text { is nondecreasing. }\right) ; \\
1-(1-\varepsilon)^{p-1} \leq \varepsilon \text { and }\left(2^{2-p}-1\right)^{1 / p} \leq 1 ; \\
L_{\varepsilon} / K_{\varepsilon} \leq \frac{\left[2-\left(1-\varepsilon_{p}\right)^{p}\right]^{1 / p}}{K_{\varepsilon}}=\left(2^{2-p}-1\right)^{1 / p} \cdot\left(\varepsilon_{p}-\varepsilon\right)-1 \leq\left(\varepsilon_{p}-\varepsilon\right)^{-1} ; \\
2^{2-p}-1 \geq(2-p) \ln 2 \geq(2-p) \cdot \frac{3}{5} .
\end{gathered}
$$

Applying these inequalities to what we have obtained in Step 2, we find that

$$
B_{p}(\varepsilon)\|f\| \leq\|T f\| \leq(1+\varepsilon)\|f\| \text { for all } f \in L^{p}(\Omega),
$$

where $B_{p}(\varepsilon)$ is given above. This completes the proof. 
Corollary 1. Let $p \in(1,2)$. Suppose that $T: L^{p}(\Omega) \rightarrow L^{p}\left(\Omega_{1}\right)$ is an i.p.c. operator. If either the range of $T$ is dense in $L^{p}\left(\Omega_{1}\right)$ or $T$ is positive, then $T$ is an isometry.

Proof. Use Propositions 2 and 3.

\section{CONCLUding REMARK}

We conclude by noting connections between the results obtained in this paper and those previously obtained on the subject of "almost isometries." By using the results of this paper, one can easily refine some of the results given in [2], [3], and [1]. For example, we have

Proposition 4. Assume the hypotheses of either Proposition 1, 2, or 3. Then there exists an isometry $U: L^{p}(\Omega) \rightarrow L^{p}\left(\Omega_{1}\right)$ such that $\|T-U\| \leq d_{p}(\varepsilon)$ and $d_{p}(0)=0$, where $d_{p}(\varepsilon)$ is a continuous function which depends only on $p$ and $\varepsilon$.

Proof. This is immediate from [1] and our three propositions.

It remains an open question as to whether one can dispense with the special hypotheses on $T$ in Propositions 2 and 3.

\section{ACKNOWLEDGMENT}

I am grateful to the referee for his valuable advice and meticulous help.

\section{REFERENCES}

1. D. E. Alspach, Small into isomorphisms on $L_{p}$ spaces, Illinois J. Math. 27 (1983), 300-314.

2. P. Enflo and H. P. Rosenthal, Some results concerning $L_{p}(\mu)$ spaces, J. Funct. Anal. 14 (1973), 325-348.

3. G. Schechtman, Almost isometric $L_{p}$ subspaces of $L_{p}(0,1)$, J. London Math. Soc. (2) 20 (1979), 516-528.

4. H. H Schaefer, Banach lattices and positive operators, Springer-Verlag, Berlin-Heidelberg-New York, 1974.

Department of Mathematics, Nankai University, Tianjin City, People's Republic OF ChINA 